\title{
EFEITOS DO AQUECIMENTO E DO ALONGAMENTO NA RESPOSTA NEUROMUSCULAR DOS ISQUIOTIBIAIS
}

\author{
EFFECTS OF WARM-UP AND STRETCHING ON THE NEUROMUSCULAR RESPONSE \\ OF THE HAMSTRINGS
}

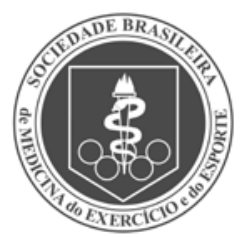

Artigo Original

\author{
EFECTOS DEL CALENTAMIENTO Y DEL ALONGAMIENTO EN LA RESPUESTA \\ NEUROMUSCULAR DE LOS ISQUIOTIBIALES
}

\begin{abstract}
João Felipe de Souza Nogueira' (Fisioterapeuta)

Caio Alano de Almeida Lins ${ }^{1}$ (Fisioterapeuta)

Angelica Vieira Cavalcanti Souza (Fisioterapeuta)

Jamilson Simões Brasileiro (Fisioterapeuta)

1. Laboratório de Análise da Performance Neuromuscular (LAPERN). Programa de Pós-Graduação em Fisioterapia. Departamento de Fisioterapia, Universidade Federal do Rio Grande do Norte (UFRN), Natal, RN, Brasil.
\end{abstract}

\section{Correspondência:}

Jamilson Simões Brasileiro, Departamento de Fisioterapia, Universidade Federal do Rio Grande do Norte, Av. Senador Salgado Filho, 3000. Campus Universitário, Lagoa Nova, 59.078-970, Natal, RN, Brasil. brasileiro@ufrnet.br

\section{RESUMO}

Introdução: A preparação para a atividade esportiva frequentemente envolve aquecimento e/ou alongamento, no intuito de prevenir lesões e/ou melhorar o desempenho. No entanto, a maneira como essas práticas podem interferir nos mecanismos neuromusculares de controle articular não é clara. Objetivo: Analisar o efeito imediato do alongamento e do aquecimento, isolados ou associados, no tempo de latência muscular (TLM) e na amplitude de ativação dos isquiotibiais, após perturbações repentinas da articulação do joelho. Métodos: Sessenta voluntários do sexo feminino (média de idade de 22,3 \pm 1,7 anos e IMC 21,4 \pm 1,3 kg/ $\mathrm{cm}^{2}$ ) foram divididas aleatoriamente em quatro grupos: (1) controle, (2) aquecimento, (3) alongamento, (4) aquecimento + alongamento. A atividade elétrica dos isquiotibiais foi captada por eletromiografia de superfície durante uma perturbação súbita do joelho. Um eletrogoniômetro detectou o início da variação angular, enquanto os eletrodos captaram o início e a amplitude da atividade muscular antes e após as intervenções. Como procedimento estatístico utilizou-se ANOVA two-way e post-hoc de Tukey, considerando-se como nível de significância $p<0,05$. Resultados: O TLM do bíceps femoral e do semitendíneo foi reduzido nos grupos 3 ( $p=0,048$ e 0,027, respectivamente) e 4 ( $p=0,026$ e 0,039, respectivamente). Não houve alteração significativa da amplitude de ativação muscular nos grupos avaliados. Conclusões: Os dados do presente estudo sugerem que, embora não tenham sido observadas alterações na amplitude eletromiográfica, o alongamento passivo e estático, utilizado de forma isolada ou associado ao aquecimento, promoveu uma redução do TLM dos isquiotibiais. Essa redução pode vir a ser um fator de proteção de lesões musculares e articulares, após uma perturbação súbita, como as que ocorrem na prática desportiva.

Palavras-chave: eletromiografia, latência de resposta, propriocepção.

\section{ABSTRACT}

Introduction: The preparation for sports activities often involves warm-up and/or stretching in order to prevent injuries and/or improve performance. However, how these practices may interfere with neuromuscular mechanisms of joint control is unclear. Objective: To analyze the immediate effect of stretching and warm-up exercises, alone or combined, on muscle latency time (MLT) and hamstrings activation amplitude after sudden knee joint disturbances. Methods: Sixty female volunteers (mean age 22.3 1.7 years, BMI $21.4 \pm 1.3 \mathrm{~kg} / \mathrm{cm}^{2}$ ) were randomly divided into four groups: (1) control, (2) warm-up, (3) stretching, (4) warm-up + stretching. Hamstrings electrical activity was recorded by surface electromyography during a sudden knee disturbance. An electrogoniometer detected the onset of angular variation, while electrodes recorded the onset and amplitude of muscle activity before and after interventions. Two-way ANOVA and post hoc Tukey were used in statistical analysis, considering a significance level of $p<0.05$. Results: The MLT of the biceps femoris and semitendinosus muscles was reduced in groups 3 ( $p=0.048$ and 0.027 , respectively), and 4 ( $p=0.026$ and 0.039 , respectively). There was no significant change in the amplitude of muscle activation amplitude in the evaluated groups. Conclusions: The results of this study suggest that although no changes in electromyographic amplitude were observed, static and passive stretching used alone or in combination with warm-up, caused a reduction in the hamstrings MLT. This reduction may prove to be a protective factor for muscle and joint injuries, after a sudden disturbance, such as those that occur in sports.

Keywords: electromyography, reaction time, proprioception.

\section{RESUMEN}

Introducción: La preparación para la actividad deportiva frecuentemente vincula calentamiento y/o alongamiento, con la intención de prevenir lesiones y/o mejorar el desempeño. Mientras tanto, la manera en cómo esas prácticas pueden interferir en los mecanismos neuromusculares de control articular no es clara. Objetivo: Analizar el efecto inmediato del alongamiento y del calentamiento, aislados o asociados, en el tiempo delatencia muscular (TLM) y en la amplitud de activación de los isquiotibiales, después de perturbaciones repentinas de la articulación de la rodilla. Métodos: Sesenta voluntarias del sexo femenino (promedio de edad de 22,3 1,7 años e IMC 21,4 \pm $1,3 \mathrm{~kg} / \mathrm{cm}^{2}$ ) fueron divididas aleatoriamente en cuatro grupos: (1) control, (2) calentamiento, (3) alongamiento, 
(4) calentamiento + alongamiento. La actividad eléctrica de los isquiotibiales fue captada por electromiografía de superficie durante una perturbación súbita de la rodilla. Un electrogoniómetro detectó el inicio de la variación angular, mientras que los electrodos captaron el inicio y la amplitud de la actividad muscular antes y después de las intervenciones. Como procedimiento estadístico se usó ANOVA two-way y post-hoc de Tukey, considerándose como nivel de significancia $p<0,05$. Resultados: El TLM del biceps femoral y del semitendíneo fue reducido en los grupos 3 ( $p=0,048$ y 0,027, respectivamente) y 4 ( $p=0,026$ y 0,039, respectivamente). No hubo alteración significativa de la amplitud de activación muscular en los grupos evaluados. Conclusiones: Los datos del presente estudio sugieren que, aunque no hayan sido observadas alteraciones en la amplitud electromiográfica, el alongamiento pasivo y estático, utilizado de forma aislada o asociado al calentamiento, promovió una reducción del TLM de los isquiotibiales. Esa reducción podría ser un factor de protección de lesiones musculares y articulares, después de una perturbación súbita, como las que ocurren en la práctica deportiva.

Palabras clave: electromiografía, latencia de respuesta, propiocepción.

\section{INTRODUÇÃO}

O alongamento e o aquecimento são práticas comumente utilizadas antes de qualquer atividade esportiva, seja ela competitiva ou recreacional, no intuito de prevenir lesões do sistema osteomioarticular. Acredita-se que o aumento da amplitude articular de movimento (ADM) decorrente de uma atividade preparatória ao exercício possa melhorar a performance e reduzir o risco de lesões em exercícios extenuantes ${ }^{1}$. No entanto, com exceção de um aumento da ADM, alguns estudos não têm encontrado evidências substanciais que sustentem o uso do alongamento e do aquecimento para aumento da performance ${ }^{1-8}$.

Um grande número de pesquisas tem sido desenvolvidas a respeito da influência do alongamento e do aquecimento nas propriedades viscoelásticas e de flexibilidade musculoesquelética9,10. Entretanto, os efeitos do alongamento e do aquecimento sobre os mecanismos neuromusculares de proteção articular ainda não estão bem esclarecidos. Mudanças agudas no comprimento, rigidez e na força da unidade miotendínea podem alterar a capacidade de detectar e responder a mudanças bruscas do meio externo. Uma unidade miotendínea mais complacente (maior maleabilidade do músculo e tecidos conectivos) em conjunto com distúrbios no tempo de ativação muscular podem alterar os tempos de reação e movimento ${ }^{4}$. Dessa forma, uma redução na acuidade proprioceptiva pode ser responsável por lesões articulares em atletas durante a prática esportiva ${ }^{11}$.

O acompanhamento proprioceptivo dos mecanoceptores miotendíneos e capso-ligamentares tem um papel significante na forma como o sistema nervoso central controla os movimentos. Estudos sobre propriocepção tendem a deter-se em habilidades individuais do senso de movimento articular, na habilidade passiva ou ativa de reproduzir uma determinada posição, ou ainda, detectar lentas variações de velocidade no posicionamento articular ${ }^{12-14}$. Para melhor se avaliar a relação entre propriocepção e resposta neuromuscular, algumas pesquisas têm se baseado em medidas da ativação eletromiográfica (EMG), após repentinas perturbações ou mudanças de posicionamento. Embora não mensure diretamente propriocepção, o tempo de latência muscular (TLM) pode ser usado para identificar alterações no sistema sensório-motor ${ }^{15}$.

Os efeitos benéficos do aquecimento pré-exercício no desemprenho atlético têm sido atribuídos principalmente ao aumento da temperatura muscular observado após o aquecimento ${ }^{16,17}$. A elevação da temperatura muscular aumenta a taxa de atividade da ATPase, o que pode promover a ativação mais rápida da fibra muscular 3,18,19. Behn et al. ${ }^{4}$ sugeriram que o aquecimento aumenta a velocidade de condução nervosa e poderia diminuir o tempo de resposta neuromuscular a um estímulo externo. Ao estudar a resposta eletromiográfica dos músculos gastrocnêmio e sóleo após aquecimento, Rosenbaum e Hennig ${ }^{20}$, perceberam diminuição no tempo de latência e na amplitude de ativação. Achados semelhantes foram expostos por Stewart et al. ${ }^{3}$ através da diminuição do valor do RMS e aumento da velocidade de condução nervosa na atividade eletromiográfica do vasto lateral após aquecimento.

Evidências recentes sugeriram que o alongamento pré-exercício pode comprometer a habilidade muscular para produção de força máxima ${ }^{5,7,8,21-23}$. De acordo com Cramer et al. ${ }^{1}$, a indução da diminuição de força pelo alongamento pode ser explicada através de alterações nos fatores neuromusculares como diminuição na ativação da unidade motora e/ou mudanças da sensibilidade reflexa. Em confronto com esses achados Evetovich et al. ${ }^{2}$ ao analisarem os efeitos do alongamento estático sobre a atividade eletromiográfica muscular, não encontraram alterações na amplitude de ativação pré e pós-alongamento.

Os músculos quadríceps femoral e ísquios-tibiais atuam de forma harmoniosa para fornecer estabilidade e funcionalidade à articulação do joelho. A ação simultânea dos músculos ao redor de uma articulação promove um maior contato entre as superfícies articulares, com consequente aumento da sua capacidade de resistir às cargas externas ${ }^{24}$. Desta forma, alterações no tempo ou na amplitude de resposta neuromuscular podem ser determinantes na prevenção ou causa de danos mioarticulares.

O objetivo do presente estudo foi analisar o efeito imediato do alongamento e do aquecimento, isolados ou combinados, na atividade eletromiográfica dos isquios-tibiais, após repentinas perturbações da articulação do joelho de sujeitos saudáveis.

\section{MÉTODOS}

Participaram do estudo 60 voluntárias do sexo feminino, com idade média de $22.3 \pm 1.7$ anos (IMC 21,4 $\pm 1,3 \mathrm{~kg} / \mathrm{cm}^{2}$ ). Todas eram saudáveis, sedentárias ou com nível de atividade apenas recreacional (não atletas, praticantes de até cinco horas semanais de exercícios), sem história de lesão mioarticulares do membro dominante nos últimos seis meses ${ }^{22}$. Além disso, as voluntárias deveriam assinalar dor zero na Escala Visual Analógica (EVA) na última semana.

As participantes foram recrutadas entre estudantes de uma universidade local, informadas sobre os objetivos da pesquisa e assinaram um termo de consentimento formal e esclarecido, de acordo com a resolução 196/96 do Conselho Nacional de Saúde. O estudo foi aprovado pelo Comitê de Ética em Pesquisa local obtendo parecer 161/2010.

Inicialmente foi realizada a avaliação inicial (pré) do TLM e amplitude de ativação dos ísquios-tibiais. Após essa análise, as voluntárias foram divididas através de sorteio em quatro grupos, com 15 participantes 
em cada. O grupo 1 foi considerado o grupo controle e realizaram a avaliação inicial, cinco minutos depois tornaram a realizar a segunda avaliação (pós), idêntica a avaliação inicial, sem serem submetidos a nenhum tipo de intervenção entre elas.

As voluntárias do grupo 2 realizaram, após a avaliação inicial, um aquecimento de cinco minutos em uma bicicleta estacionaria (Ergo 167 Cycle - Ergo Fit ${ }^{\circledR}$, Alemanha) com uma carga constante de 70W e uma velocidade controlada em $20 \mathrm{~km} / \mathrm{h}$; os do grupo 3 submeteram-se ao alongamento, passivo e estático, dos músculos quadríceps femoral e ísquios tibiais (três séries de 30 segundos para cada músculo, com intervalo de 30 segundos entre um alongamento e outro). Por fim, os indivíduos do Grupo 4 submeteram-se ao aquecimento de cinco minutos em bicicleta estacionária (carga de 70W e velocidade de $20 \mathrm{~km} / \mathrm{h}$ ) seguido de alongamento realizado de forma idêntica aos voluntários do grupo 3. Logo após a intervenção, os indivíduos dos grupos 2, 3 e 4 realizaram a segunda avaliação, idêntica a avaliação inicial.

A captação do sinal eletromiográfico foi realizada por meio de um Módulo Condicionador de sinais de oito canais (CS 800 - EMG System do Brasil Ltda ${ }^{\circledR}$ - São Paulo, Brasil) com resolução de 12 bits e razão de rejeição de modo comum $>80 \mathrm{~dB}$, frequência de amostragem de $1.000 \mathrm{~Hz}$, ganho total de 2.000 vezes (20 vezes no sensor e 100 vezes no EMG), filtro passa alta em $20 \mathrm{~Hz}$ e filtro passa baixa em $500 \mathrm{~Hz}$, interfaciado com um microcomputador Pentium, que recebeu o sinal eletromiográfico e o armazenou em arquivo, além de um software para análise digital de sinais, AqDados (versão 5.0). Foram utilizados para a captação da atividade elétrica dos músculos, eletrodos ativos de superfície (EMG System do Brasil Ltda ${ }^{\circledR}$ - São Paulo, Brasil), compostos por duas barras paralelas retangulares de $\mathrm{Ag} / \mathrm{AgCl}$ (com distância intereletrodo de $1 \mathrm{~cm}$ ).

Para o registro da atividade eletromiográfica (tempo de latência muscular e amplitude de ativação) dos músculos isquiotibiais, as voluntárias foram submetidas à preparação da pele com tricotomia e limpeza da área com álcool 70\%. Os eletrodos foram posicionados nos músculos bíceps femoral (BF) e semitendinoso (ST) e fixados com esparadrapo e uma faixa elástica, para evitar o deslocamento dos eletrodos. Seguindo os critérios do SENIAM ${ }^{25}$ para o posicionamento dos eletrodos, no músculo BF foi posicionado a 50\% de distância entre a tuberosidade isquiática e o epicôndilo lateral da tíbia enquanto para o músculo ST foi posicionado a 50\% da distância entre a tuberosidade isquiática e o epicôndilo medial da tíbia. O eletrodo de referência, por sua vez, foi posicionado no maléolo medial do membro contralateral.

A captação do sinal eletromiográfico do tempo de latência muscular foi realizada com a voluntária posicionada em decúbito ventral sobre uma maca especialmente adaptada (com uma haste em uma das suas extremidades), com o joelho do membro inferior dominante fletido (110 flexão) e preso por um cabo fixado no terço inferior da perna através de uma cinta, presa por sua outra extremidade na haste da maca. O eletrogoniômetro (EMG System do Brasil Ltda - São Paulo, Brasil), conectado ao eletromiógrafo, foi fixado no joelho avaliado através de tiras de velcro, com um dos seus braços paralelo ao fêmur e o outro paralelo à fíbula (figura 1).

Cada indivíduo foi esclarecido a respeito do gesto a ser realizado durante a captação do sinal (extensão do joelho dominante) e orientado a começar o movimento quando o comando verbal lhe fosse dado. Após isso o eletromiógrafo foi acionado, enquanto o voluntário tentava estender a perna avaliada com força máxima e a resistência imposta pelo cabo preso à haste era liberada abruptamente, promovendo uma mudança inesperada na angulação do joelho do indivíduo. Enquanto o eletrogoniômetro detectava o início da variação da angulação da articulação, os eletrodos do eletromiógrafo, posicionados nos respectivos pontos motores do bíceps femoral e do semitendinoso, captavam o

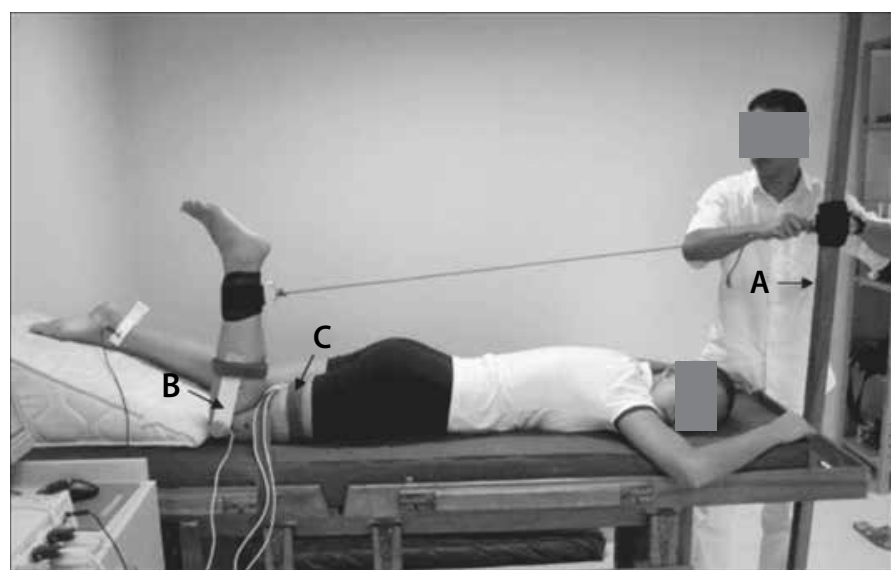

Figura 1. A) Maca com uma haste em uma de suas extremidades; B) Eletrogoniômetro posicionado na articulação do joelho ; C) Eletrodos para captação do sinal EMG.

início e a amplitude da atividade muscular, na tentativa de desacelerar a extensão do membro avaliado. Cabe ressaltar que os eletrodos não foram retirados entre as avaliações inicial e final.

\section{Tempo de latência muscular (TLM) e amplitude de ativação dos isquiotibiais}

Os valores de amplitude de ativação muscular foram dados através da Root Mean Square (RMS), em $\mu \mathrm{V}$, captados num intervalo de tempo de cinco segundos. O TLM foi considerado o espaço de tempo (em ms) decorrido entre o momento de início da variação angular ( $\geq 3$ graus para extensão do joelho), captada pelo eletrogoniômetro, e o momento de registro de uma atividade muscular efetiva, captado pelo eletrodo do eletromiógrafo.

\section{Análise Estatística}

Os dados foram analisados através do software SPSS versão 14.0. A normalização dos dados foi verificada pelo teste de Shapiro-Wilk. A comparação do TLM e a amplitude de ativação dos ísquios-tibiais foi avaliada pré e pós intervenção para cada grupo e intergrupos através da ANOVA two-way, optando-se pelo teste post-hoc de Tukey quando for observada diferença entre os grupos. Em todas as situações foi considerado um $\mathrm{p} \leq 0,05$ como significativo.

\section{RESULTADOS}

Os resultados revelaram que não houve mudança significativa ( $p>0.05$ ) na amplitude de ativação em nenhum dos grupos de intervenção quando comparado os valores de RMS normalizado antes e após o aquecimento e/ou alongamento, para os músculos bíceps femoral e semitendinoso. Também não houve diferença significante ( $p>0.05$ ) nos valores de RMS entre os grupos avaliados, para nenhum dos músculos (figura 2).

O TLM foi significativamente reduzido do pré para o pós-teste nos grupos 3 e 4 ( $p<0.05)$ para os músculos bíceps femoral e semitendinoso; entretanto não se observou mudanças significativas dentro dos grupos 1 e 2. Quando comparado ao grupo controle (1), os grupos 3 e 4 apresentaram uma redução significativa $(p<0.05)$ no TLM do bíceps femoral e do semitendinoso. Os grupos 3 e 4 apresentaram uma redução significativa no TLM quando comparado ao grupo 2, no entanto, sem diferença significativa entre eles (figura 3).

\section{DISCUSSÃO}

Os resultados do presente estudo indicaram que o alongamento passivo e estático, realizado de forma isolada ou associada ao aquecimento, diminuiu o TLM dos ísquios-tibiais. Achados conflitantes aos 


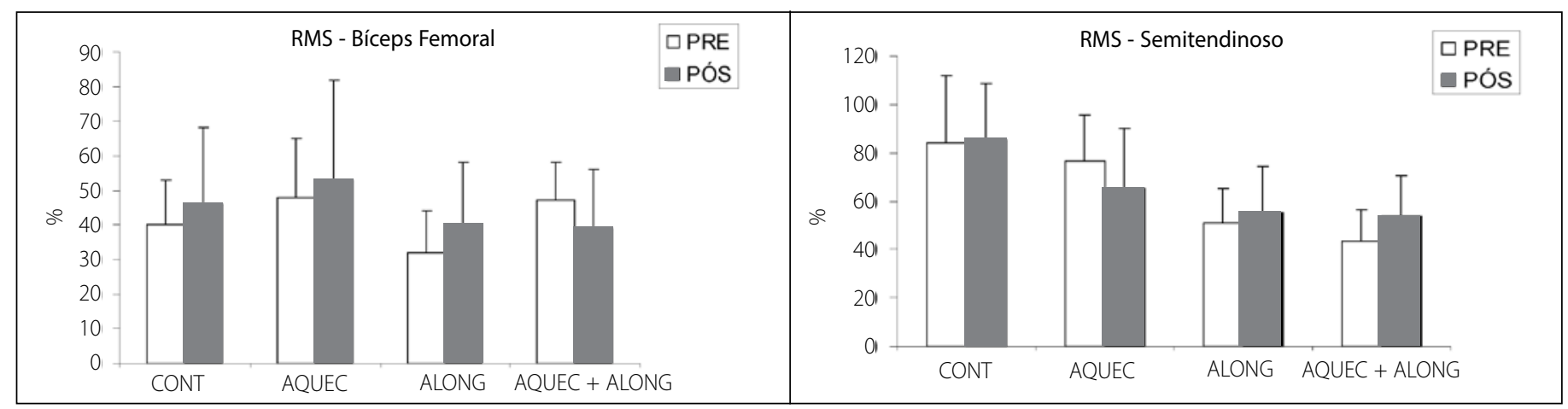

Figura 2. RMS antes e após intervenção em cada grupo (Controle; Aquecimento; Alongamento; Aquecimento + Alongamento).

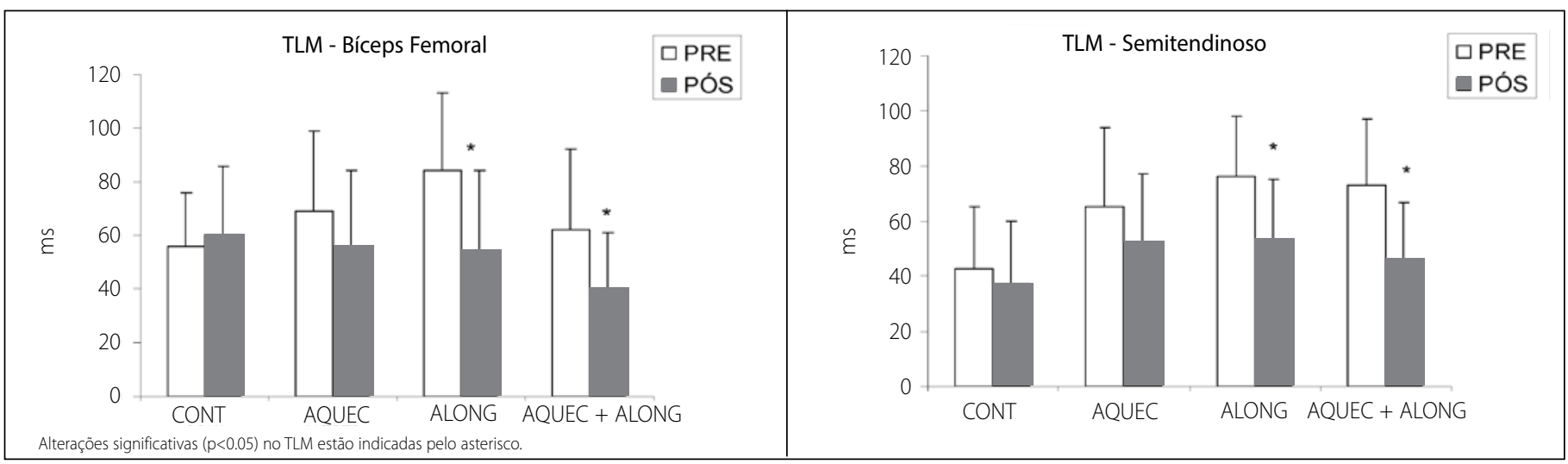

Figura 3. TLM antes e após intervenção em cada grupo (Controle; Aquecimento; Alongamento; Aquecimento + Alongamento).

anteriormente citados foram publicados por Rosenbaum e Hennig ${ }^{20}$, que perceberam, através de análise eletromiográfica, um aumento no tempo de latência dos músculos gastrocnêmio e sóleo, após a realização do alongamento passivo.

Assim, o efeito imediato do alongamento sobre o TLM parece ser músculo específico e depende da função e estrutura anatômica do grupo muscular avaliado'. Os ísquios-tibiais atuam de forma sinérgica com o LCA evitando a translação anterior da tíbia e, particularmente nesse caso, a redução no tempo de ativação muscular poderia auxiliar na prevenção de danos articulares. Sugere-se então, que o alongamento passivo e estático possa promover alterações no feedback proprioceptivo dos músculos posteriores do joelho a partir da pré-ativação dos proprioceptores mecânicos de baixo limiar que colocariam o fuso muscular em pronto estado de ação ${ }^{26}$.

O aquecimento, quando realizado de forma isolada, promoveu uma diminuição no TLM, mas esta diminuição não foi significante. Stewart et al. ${ }^{3}$, encontraram uma diminuição significante da latência do músculo vasto lateral após 15 minutos de aquecimento no cicloergômetro a 70\% do limiar anaeróbio. Assim, uma razão para a diminuição do TLM não ter atingido índices de significância pode ter sido o curto tempo de aquecimento realizado neste experimento. Dessa forma o aumento da temperatura muscular não ocorreria de forma suficiente para promover uma alteração na temperatura neural o que resultaria em uma maior velocidade de condução nervosa e, consequentemente, em um menor tempo de latência muscular.

Os resultados do atual estudo não mostraram alteração em nenhum dos grupos, no que se refere à amplitude de ativação eletromiográfica. Muitas pesquisas avaliaram a amplitude de ativação neuromuscular após um aquecimento ou alongamento e resultados diversos têm sido encontrados. Fatores metodológicos na captação da atividade eletromiográfica, tais como tipo e velocidade de contração, e até a função muscular do grupo avaliado, parecem influenciar na resposta. Cramer et al. ${ }^{6}$ não perceberam mudanças na amplitude eletromiográfica do quadríceps, após um protocolo de alongamento estático, captada durante uma contração excêntrica isocinética. Esses achados corroboram com os do presente estudo, no qual a intensidade da atividade neuromuscular dos ísquios-tibiais durante uma atividade predominantemente excêntrica não foi alterada. No entanto, a maioria dos estudos 1,20,22 que avaliaram o efeito imediato do alongamento na amplitude eletromiográfica, realizaram uma contração concêntrica ou isométrica e observaram uma diminuição na ativação muscular.

Grande parte dos estudos com EMG de superfície tem avaliado o comportamento do quadríceps femoral e talvez, devido a isso, o comportamento diferenciado e específico de cada grupo muscular não venha sendo observado. Evetovich et al. ${ }^{2}$ não encontraram diferenças na amplitude de ativação do músculo bíceps braquial durante uma contração concêntrica isocinética, após o alongamento. Eles atribuíram esses achados a especificidade anatômica e funcional do bíceps braquial, que atua como desacelerador da extensão do cotovelo. De forma semelhante, nossos resultados demonstraram que o alongamento não modificou a amplitude EMG dos ísquios-tibiais. A opção de avaliar a atividade excêntrica deste grupo muscular deveu-se a importância desse gesto na fase de desaceleração do joelho, movimento realizado na execução de diversos gestos esportivos.

Diferente dos nossos resultados, Stewart et al. ${ }^{3}$ perceberam uma diminuição significante da amplitude EMG do quadríceps femoral em voluntários submetidos a 15 minutos de aquecimento na esteira. Eles atribuíram esse decréscimo ao aumento da velocidade de abertura e fechamento dos canais dos íons de sódio no músculo, decorrente do aumento da temperatura. A redução do tempo de difusão dos íons de sódio para dentro da célula provocaria uma diminuição na amplitude do potencial de ação, que se traduziria em uma diminuição da amplitude $\mathrm{EMG}^{27}$. Tal efeito fisiológico, talvez não tenha se evidenciado no presente estudo pelo fato do curto tempo de aquecimento não promover uma alteração térmica muscular suficiente.

Os alongamentos estático e passivo realizado em nível de descon- 
forto nas voluntárias deste estudo parecem modificar as propriedades neuromusculares dos músculos que agem sobre o joelho. A maneira como o alongamento pode promover alterações preparatórias e contribuir para uma resposta proprioceptiva mais eficaz ainda não foram claramente estabelecidas.

\section{CONCLUSÃO}

Os dados do presente estudo sugerem que, embora não tenham sido observadas alterações na amplitude eletromiográfica, o alongamento, utilizado de forma isolada ou associado ao aquecimento, pro- moveu uma redução do tempo de latência muscular dos ísquios-tibiais. Essa redução pode vir a ser um fator de proteção de lesões musculares e articulares, após uma súbita perturbação, como aquelas realizadas na prática desportiva. Futuros estudos devem considerar o comportamento neuromuscular em diferentes tipos de contração, bem como diferentes velocidades angulares.

Todos os autores declararam não haver qualquer potencial conflito de interesses referente a este artigo.

\section{REFERÊNCIAS}

1. Cramer JT, Housh TJ, Johnson GO, Weir JP, Coburn JW, Beck TW. The acute effects of static stretching on peak torque, mean power output, electromyography, and mechanomyography. Eur J Appl Physiol. 2005;93:530-9.

2. Evetovich TK, Nauman NJ, Conley DS, Todd JB. Effect of static stretching of the biceps brachii on torque, electromyography,and mechanomyography during concentric isokinetic muscle actions. J Strength Cond Res. 2003;17:484-8.

3. Stewart D, Macaluso A, De Vito G. The effect of an active warm-up on surface EMG and muscle performance in healthy humans. Eur J Appl Physiol. 2003;89:509-13.

4. Behm DG, Bambury A, Cahill F, Power K. Effect of Acute Static Stretching on Force, Balance, Reaction Time, and Movement Time. Med Sci Sports Exerc. 2004;36:1397-402.

5. Cramer JT, Housh TJ, Johnson GO, Miller JM, Coburn JW, Beck TW. The acute effects of static stretching on peak torque of the stretched and unstretched limbs in women. J Strength Cond Res. 2004;18:236-41.

6. Cramer JT, Housh TJ, Johnson GO, Weir JP, Beck TW, Coburn JW. An acute bout of static stretching does not affect maximal eccentric isokinetic peak torque, the joint angle at peak torque, mean power, electromyography, or mechanomyography. J Orthop Sports Phys Ther. 2007b;37:130-9.

7. Bacurau RF, Monteiro GA, Ugrinowitsch C, Tricoli V, Cabral LF, Aoki MS. Acute effect of a ballistic and a static stretching exercise bout on flexibility and maximal strength. J Strength Cond Res 2009;23:304-8

8. Herda TJ, Ryan ED, Costa PB, Walter AA, Hoge KM, Uribe BP, et al. Acute effects of passive stretching and vibration on the elctromechanical delay and musculotendinous stiffness of the plantar flexors. Electromyogr Clin Neurophysiol. 2010;50:137-48.

9. Vieira WHB, Valente RZ, Greve JMA, Brasileiro JS. Efeitos de duas técnicas de alongamento muscular dos ísquios-tibiais na amplitude de extensão ativa do joelho e no pico de torque. Rev Bras Fisioter. 2005;9:71-6.

10. Brasileiro JS, Faria A, Queiroz LL. Influência do Resfriamento e do Aquecimento na Flexibilidade dos Músculos ísquios-tibiais. Rev Bras Fisioter. 2007;11:01-5.

11. Pincivero DM, Lephart SM, Sterner $R$. The effects of muscular fatigue on shoulder proprioception. Clin J Sport Med. 1998;8:96-101.

12. Larsen R, Lund H, Christensen R, Rogind H, Danneskiold-Samsøe B, Bliddal H. Effect of static stretching of quadriceps and hamstring muscles on knee joint position sense. Br J Sports Med. 2005;39:43-6.

13. Bjo" rklund M, Djupsjo" backa M, Crenshaw AG. Acute Muscle Stretching and shoulder Position Sense. J Athl Train. 2006;41:270-4.
14. Brindle TJ, Nitz AJ, Uhl TL ,Kifer E, Shapiro R. Kinematic and EMG characteristics of simple shoulder movements with proprioception and visual feedback. J Electromyogr Kinesiol. 2006;16:236-49.

15. Brindle TJ, Nyland JA, Nitz AJ, Shapiro R. Scapulothoracic latent muscle reaction timing comparison between trained overhead throwers and untrained control subjects. Scand J Med Sci Sports. 2007;17:252-9.

16. Young $W$, Behm D. Should static stretching be used during a warm-up for strength and power activities? Strength Cond J. 2002;24:33-7.

17. Young WB. The use of static stretching in warm-up for training and competition. Int J Sports Physiol Perform. 2007;2:212-6

18. Bishop D. Warm up I: potential mechanisms and the effects of passive warm up on exercise performance. Sports Med. 2003a;33:439-54.

19. Bishop D. Warm up II: performance changes following active warm up and how to structure the warm up. Sports Med. 2003b;33:483-98.

20. Rosenbaum D, Hennig EM. The influence of stretching and warm-up exercises on Achilles tendon reflex activity. J Sports Sci. 1995;13:481-90.

21. Behm DG, Button DC, Butt JC. Factors affecting force loss with prolonged stretching. Can J Appl Physiol 2001;26:261-72.

22. Cramer JT, Beck TW, Housh TJ, Massey LL, Marek SM, Danglemeier S, et al. Acute effects of static stretching on characteristics of the isokinetic angle-torque relationship, surface electromyography, and mechanomyography. J Sports Sci. 2007a;25:687-98.

23. Ogura Y, Miyahara Y, Naito H, Katamoto S, Aoki J (2007) Duration of static stretching influences muscle force production in hamstring muscles. J Strength Cond Res. 2007;21:788-92.

24. Aquino CF, Viana SO, Fonseca ST, Bricio RS, Vaz DV. Mecanismos neuromusculares de controle da estabilidade articular. R. Bras. Ci e Mov. 2004;12:35-42.

25. Hermens HJ, Freriks B, Disselhorst-Klug C, Rau G. Development of recommendations for SEMG sensors and sensor placement procedures. J Electromyogr Kinesiol. 2000;10(5):361-74.

26. Shultz SJ, Perrin DH, Adams JM, Arnold BL, Gansneder BM, Granata KP. Neuromuscular Response Characteristics in Men and Women After Knee Perturbation in a Single-Leg, Weight-Bearing Stance. J Athl Train. 2001;36:37-43

27. Rutkove SB. Effects of temperature on neuromuscular electrophysiology. Muscle Nerve. 2001;24:867-82 